\title{
Guidance Scheme for Modulation of Drag Devices to Enable Return from Low Earth Orbit
}

\author{
Soumyo Dutta, ${ }^{*}$ Angela L. Bowes, ${ }^{*}$ Alicia D. Cianciolo, ${ }^{*}$ and Christopher E. Glass, ${ }^{\dagger}$ \\ NASA Langley Research Center, Hampton, VA 23681, USA \\ Richard W. Powell ${ }^{\ddagger}$ \\ Analytical Mechanics Associates, Hampton, VA 23681, USA
}

\begin{abstract}
Passive drag devices provide opportunities to return payloads from low Earth orbits quickly without using onboard propulsive systems to de-orbit the spacecraft. However, one potential disadvantage of such systems has been the lack of landing accuracy. Drag modulation or changing the shape of the drag device during flight offer a way to control the de-orbit trajectory and target a specific landing location. This paper discusses a candidate passive drag based system, called Exo-brake, as well as efforts to model the dynamics of the vehicle as it de-orbits and guidance schemes used to control the trajectory. Such systems can enable quick return of payloads from low Earth orbit assets like the International Space Station without the use of large re-entry cargo capsules or propulsive systems.
\end{abstract}

\section{Introduction}

Since the retirement of the Space Shuttles, there has been an increased interest in technologies that can return payloads from assets in low Earth orbits, such as the International Space Station (ISS). Several commercial ventures have proposed large re-entry capsules that will bring back large amount of payloads; however, these capsules are launched infrequently, are cost prohibitive, and are impractical to return small amounts of payloads (less than $100 \mathrm{~kg}$ ) from places like the ISS. There have been proposals to develop a Small Payload Quick Return (SPQR) class of vehicles to meet the demands of returning small amounts of payloads from the ISS. One of these proposed devices is the Exo-brake, depicted in Fig. 1, that consists of a parachute-like device designed to produce drag in exoatmospheric conditions. ${ }^{1-3}$ The Exo-brake device has been successfully demonstrated in flights from the ISS in 2014 and $2015 .{ }^{3}$ Additional demonstration flights are expected in 2017.

In the flights from the ISS through 2015, the Exo-brake used a constant shape during exoatmospheric flight and included no re-entry vehicle. However, future missions plan to demonstrate modulation of the drag shape during exoatmospheric flight. The hypothesis for the drag modulation demonstration is that the vehicle can generate enough differential force to change its trajectory and achieve pre-determined landing targets. The payload return concept of operation pairs the Exo-brake with an entry vehicle, like a Tube Deployed Reentry Vehicle (TDRV) or a Reentry Breakup Recorded (REBR) class vehicle (pictured in Fig. 2). In this configuration, the Exo-brake is jettisoned at approximately $90 \mathrm{~km}$ altitude and the re-entry vehicle allows a payload to survive atmospheric entry and land successfully at a pre-determined location.

This paper discusses the efforts to model the flight dynamics of an Exo-brake paired with a REBR vehicle and the development of a guidance scheme to demonstrate the feasibility of drag modulation as a mean of targeting landing locations. A comparison of the simulation results and past Exo-brake mission flight data are shown. Finally, performance of the simulation and guidance scheme is demonstrated by analyzing a test problem of landing within a $50 \mathrm{~km}$ radius at Utah Test and Training Range (UTTR).

\footnotetext{
*Aerospace Engineer, Atmospheric Flight and Entry Systems Branch, 1 N. Dryden St., MS 489, and AIAA Member.

$\dagger$ Aerospace Research Engineer, Aerothermodynamics Branch, MS 408A, and Senior AIAA Member

$¥$ Aerospace Engineer, Analytical Mechanics Associates, Atmospheric Flight and Entry Systems Branch, 1 N. Dryden St., MS 489, and AIAA Associate Fellow.
} 


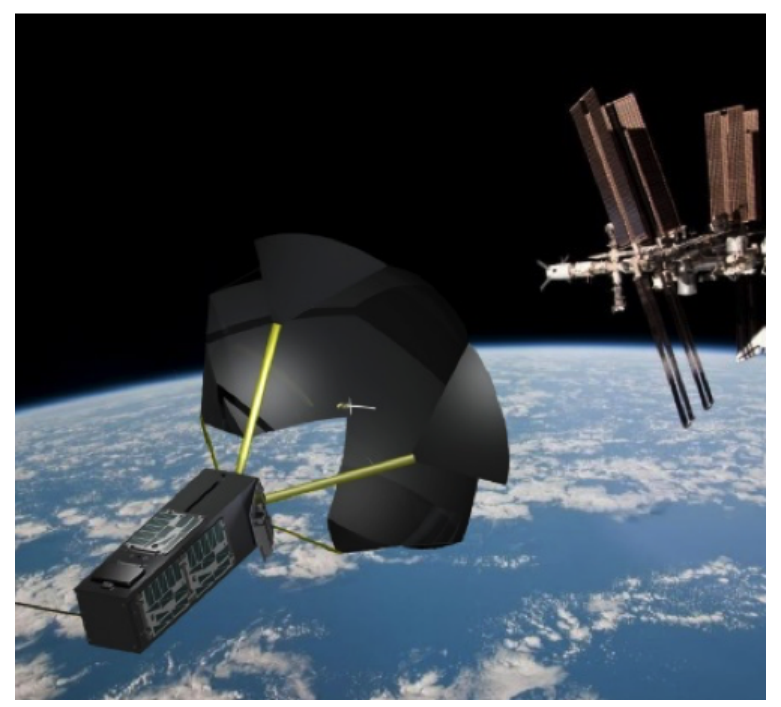

Figure 1. Artist conception of the passive drag based device, Exo-brake, with a cubesat payload jettisoning from the International Space Station. ${ }^{3}$

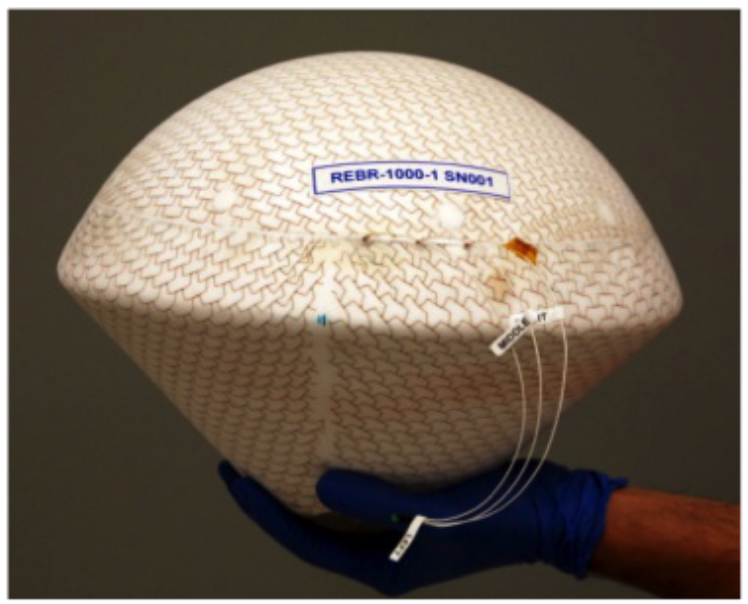

Figure 2. Reentry Breakup Recorder used on the return from ISS flight. ${ }^{4}$

\section{Simulation Detail}

The Program to Optimize Simulated Trajectories II (POST2) is the tool used for the end-to-end (ISS to Earth surface) simulation of the exoatmospheric and atmospheric re-entry flight of the Exo-brake and REBR vehicles. POST2 is a generalized point mass, rigid body, discrete parameter targeting and optimization trajectory simulation program based on the POST software initially developed in the 1970's by NASA Langley Research Center in partnership with the Martin Marietta Co. to support Space Shuttle development. POST2 is a six degree-of-freedom flight dynamics simulation tool that can simultaneously simulate the trajectory of up to 20 independent or connected rigid bodies. The software has been used successfully for several Entry, Descent, and Landing (EDL) missions, such as Mars Pathfinder, ${ }^{5}$ Mars Exploration Rovers, ${ }^{6}$ Stardust Earth Entry Capsule, ${ }^{7}$ Mars Phoenix,${ }^{8}$ and Mars Science Laboratory. ${ }^{9}$ The NASA Langley Research Center POST2 has been integrated with engineering models of the atmosphere, such as the Earth Global Reference Atmospheric Model (GRAM), and can interact with high fidelity aerodynamic databases. The Exo-brake simulation developed with POST2 has been used to match flight data from past Exo-brake missions and serves as a test bed to develop guidance schemes for future missions where Exo-brake will be modulated to control its trajectory. The simulation could be used to determine the correct call rate schedule, latencies, and 
necessary navigation and controller schemes necessary to operate the Exo-brake. However, for this study, only the prototype of a guidance scheme is tested with this flight simulation. Other details, such as latencies in the system, controller and actual interactions etc., that must be considered for a high-fidelity simulation of the flight are not considered here.

The Exo-brake can be modeled as a 6-degree-of-freedom system with an aerodynamic database that provides axial force, normal force, side force, pitching moment, yawing moment, and rolling moment coefficients. These aerodynamic properties are a function of the vehicle angle of attack, sideslip angle, Knudsen number, and percentage of the Exo-brake that is deployed (called deployment level). However, for this study and development of a prototype guidance scheme, the vehicle's attitude has been constrained to angle of attack and sideslip angles of 0 degrees. Thus, the Exo-brake has been effectively modeled as a 3-degree-of-freedom vehicle for this study. It is theorized that the Exo-brake will not demonstrate large-scale attitude dynamics during its actual flight, thus, to the first order, the constrained attitude assumption is valid.

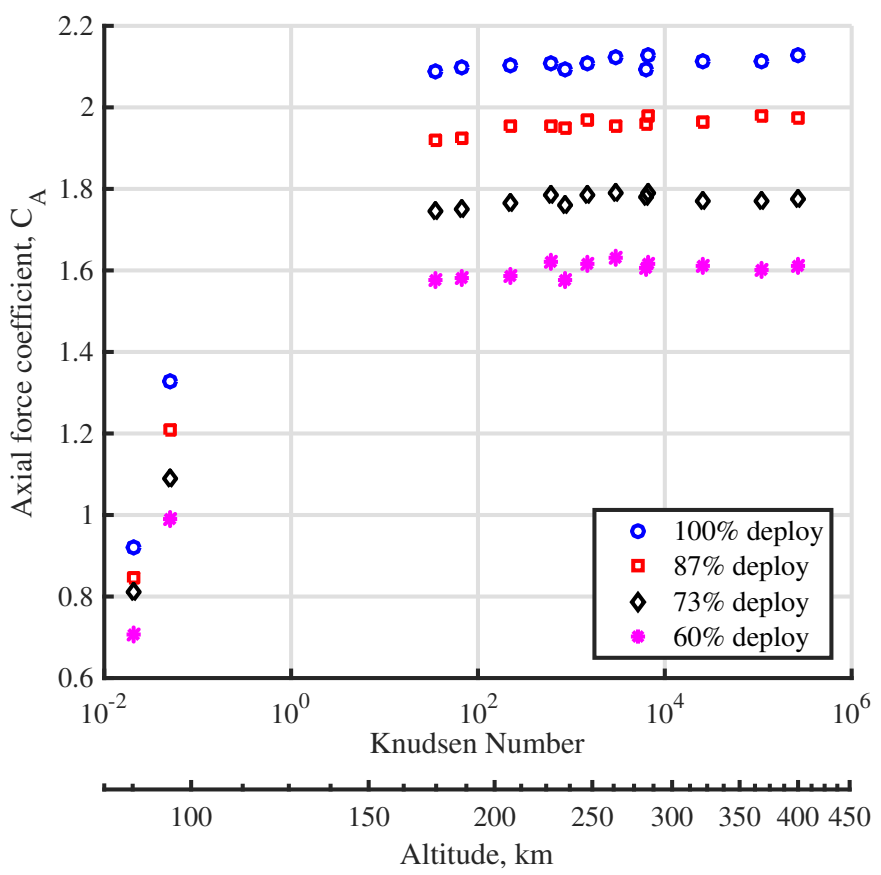

Figure 3. Axial force coefficient of the Exo-brake shape as a function of Knudsen number. Coefficients are non-dimensionalized based on the reference area of the fully deployed shape.

Since vehicle movement is restricted, the only aerodynamic force on the body is the the axial force. The axial force coefficients for various deployment levels are shown in Fig. 3. The various deployment levels are illustrated in Fig. 4. When the Exo-brake is modulated from the $100 \%$ (or fully deployed) shape, only the horizontal arms are retracted. Moreover, due to manufacturing and design restrictions assumed for this study, the horizontal arm can be only retracted up to $60 \%$ of its initial length. This position is referred to as the $60 \%$ deployed case, even though the projected, reference area of this shape is $76 \%$ of the fully deployed reference area. Fig. 4 shows other possible deployed shapes between the fully deployed and maximum retracted states, although it should be noted that Exo-brake is not restricted to only these deployment levels. The axial force coefficients in Fig. 3 have been non-dimensionalized using the reference area of the fully deployed case and show the expected pattern of lower axial force coefficients when the Exo-brake has been retracted. It is also noted that the axial force coefficient appears to be nearly constant at high Knudsen numbers, which correspond to rarefied atmosphere seen near $300-400 \mathrm{~km}$ altitudes, while the axial force coefficient decreases significantly for lower Knudsen numbers associated with the continuum flight regime near $90 \mathrm{~km}$ altitude. The vehicle does not spend considerable time in altitudes between 100 and $150 \mathrm{~km}$, since by that time the vehicle is descending and not in a complete orbit, so the aerodatabase is currently sparse in those altitude regimes. Future work will add aerodynamic data in those regions as well.

The simulation transitions to the REBR spacecraft mass properties and aerodynamics at $90 \mathrm{~km}$ altitude. Currently the separation dynamics are not modeled and the transition occurs instantaneously. However, the 


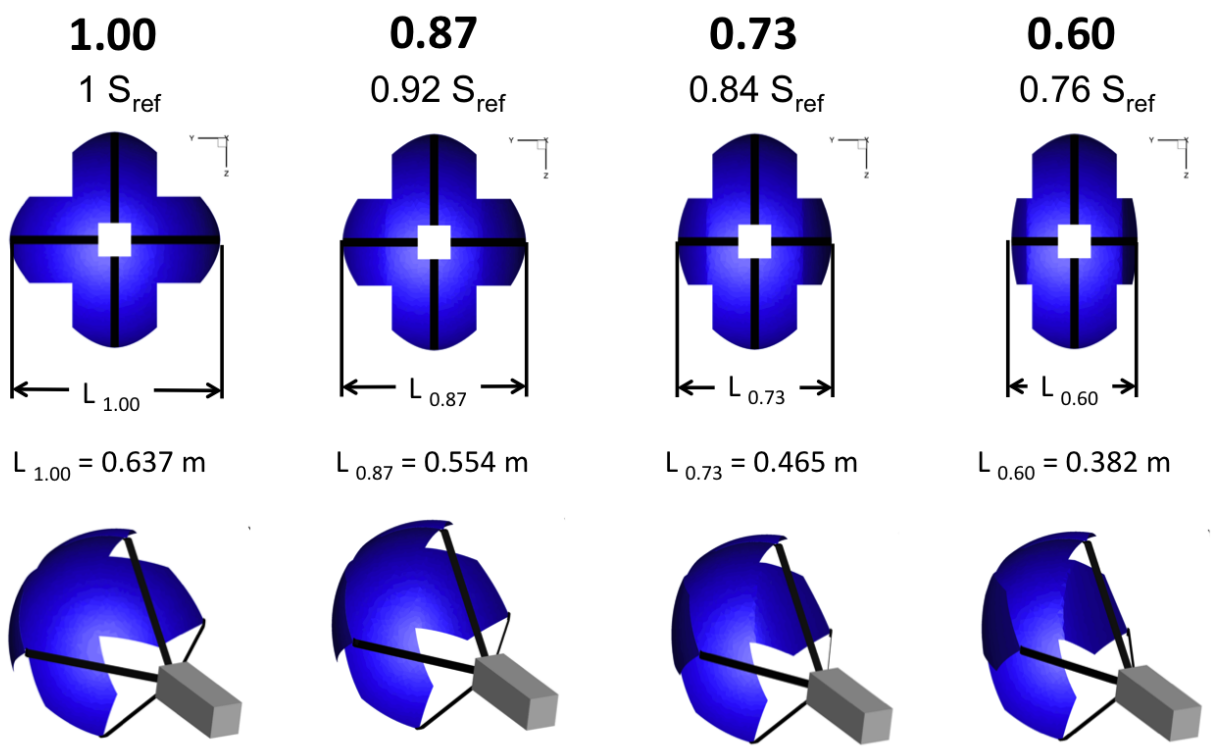

Figure 4. Various potential deployed states of the Exo-brake.

Exo-brake end states do become the REBR entry states, thus maintaining end-to-end trajectory properties. The REBR mass properties are derived from past de-orbit missions from the ISS $^{4}$ and the aerodynamics is based on the six degree-of-freedom aerodynamic database developed for the Mars Microprobe vehicle ${ }^{10}$ whose 45 degree sphere-cone with a spherical backshell shape has been replicated by the REBR vehicle. The REBR is simulated to fly a ballistic trajectory during entry.

This analysis does not consider structural and thermal integrity of the Exo-brake at $90 \mathrm{~km}$ altitude. Parallel work is being conducted to determine the minimum altitude at which the Exo-brake can survive. If that minimum altitude is greater than $90 \mathrm{~km}$, the transition to the re-entry capsule - REBR or some other device - will occur well above $90 \mathrm{~km}$. However, the results of this analysis are still transferable to such a modified concept of operations.

The atmospheric model used for continuum flow regime (approximately $90 \mathrm{~km}$ altitude and below) is the Earth Global Reference Atmosphere Model 2010 (Earth GRAM 2010) ${ }^{11}$ which models both nominal and offnominal atmospheric conditions. For the thermosphere portion (altitudes above $90 \mathrm{~km}$ ) of the flight, GRAM provides three options: Marshall Engineering Thermospheric (MET) model, the Naval Research Laboratory's Mass Spectrometer and Incoherent Scatter Radar (MSIS) 90 model, and the 2008 Jacchia-Bowman (JB2008) Thermospheric model. Results from all three models are strongly determined by a handful of thermospheric parameters, such as the Solar heat flux at $10.7 \mathrm{~cm}$ wavelength (commonly referred to as F10.7), the 81-day moving average of the F10.7 quantity, and the geomagnetic index (often referred to as AP index). Ultimately, the first model was chosen as the thermospheric model for the simulation because it most closely matched the past Exo-brake flight data. These reconstructions results are discussed in more detail in later sections.

Finally, Monte Carlo analysis was performed with the simulation to gauge the robustness of the guidance schemes developed to target the landing location. The Monte Carlo simulation varied the atmospheric conditions - density, pressure, temperature, speed of sound, mean freepath, and winds - based on GRAM's internal random number process and the REBR aerodynamic properties based on the six degree-of-freedom aerodatabase. The Exo-brake aerodynamic uncertainties were not quantified at the time of this study and hence the nominal axial force coefficient was used. Additionally, mass properties and initial states were not varied in the current results presented in the paper, but further development of the guidance schemes will also test robustness to those dispersed conditions.

The concept of operations of the current design problem is shown in Fig. 5. The Exo-brake vehicle is initialized from ISS orbital states and the Exo-brake shape is deployed. Initially, for approximately the first five days, the vehicle holds the $100 \%$ deployed shape to quickly traverse the high altitude regime of the flight where the dynamic pressure (and hence controllability) of the vehicle is low. Then a predictor-corrector guidance scheme determines the starting time and deployment levels for various phases of flight when the 
deployment level is held constant. The guidance scheme propagates the trajectory to the ground and then optimizes the deployment times and levels to target a $50 \mathrm{~km}$ circle at UTTR. Finally, at $90 \mathrm{~km}$ altitude, vehicle properties are transitioned to the REBR shape and the final atmospheric EDL portion is simulated.

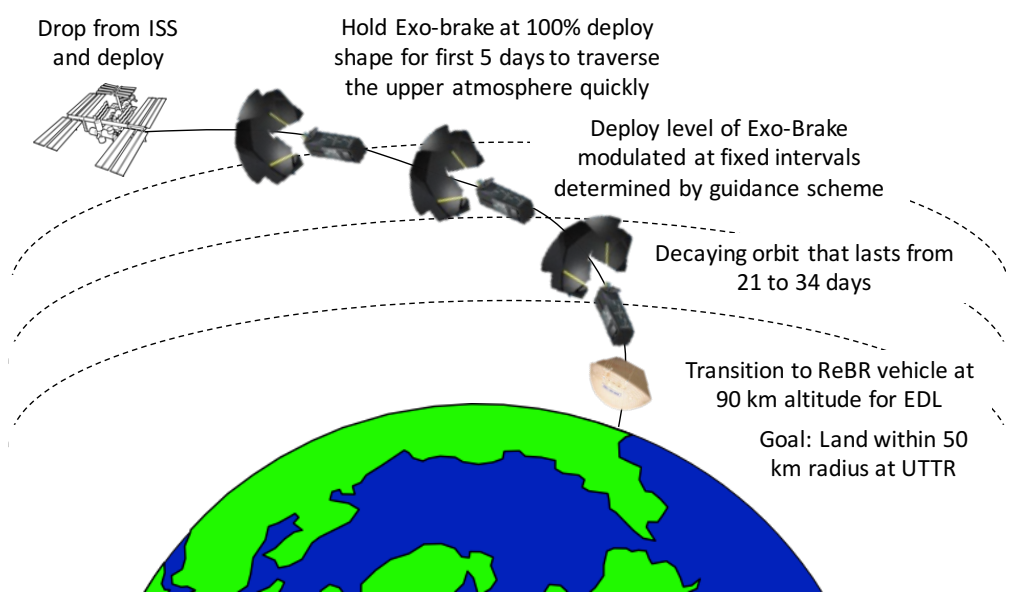

Figure 5. Concept of operations of the Exo-brake and REBR de-orbit trajectory from the ISS.

\section{Results}

\section{A. Reconstruction of Past Exo-brake Demonstrations}

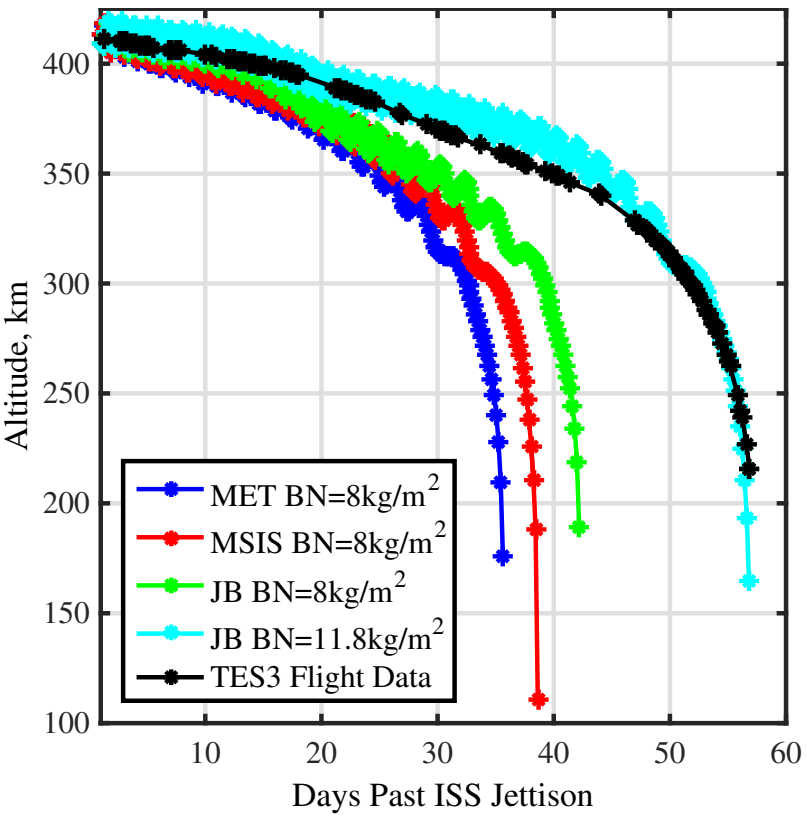

(a) TES3 Reconstruction (2014 flight)

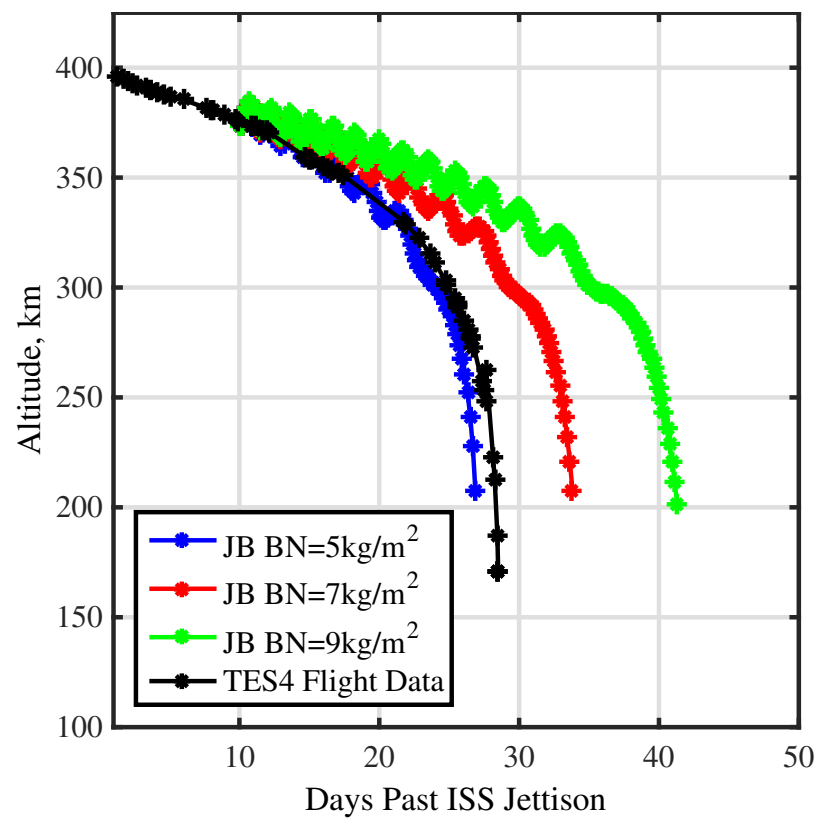

(b) TES4 Reconstruction (2015 flight)

Figure 6. Reconstruction of the past Exo-brake demonstrations with the POST2 flight dynamics simulation.

Development of the Exo-brake simulation greatly benefited from two past missions in 2014 and 2015 that provided trajectory data after the Exo-brake was jettisoned from the ISS. ${ }^{3}$ As the simulation was developed, several key decisions, such as the thermosphere model selection, were based on the parameters that best reconciled the simulation with the the flight data. Unfortunately, these past vehicles did not have onboard sensors, such as inertial measurement units or Global Positioning System (GPS) receivers. The flight data 
were in the form of Two-line Elements (TLE) and based on ground range estimates of the spacecraft taken every few days. Thus, the data was coarse and attitude reconciliation between the simulation and the flight data was not possible. Additionally, since the data was in the form of TLE at large increments of time, the data appeared to be smoother than the variation in the orbital altitude expected due to the effect of oblateness in Earth's gravity.

Figure 6 shows the flight data from the past two Exo-brake flights that were part of the TechEd Sat (TES) 3 and 4 missions $^{3}$ along with reconciled predictions from the simulation. An Exo-brake aerodynamic database had not been generated in time for these two missions, so the ballistic number (BN), which is mass divided by the drag coefficient and reference area, were unknown. For TES3, the pre-flight ballistic number was estimated to be $8 \mathrm{~kg} / \mathrm{m}^{2} \pm 3 \mathrm{~kg} / \mathrm{m}^{2}$, while for TES4, the ballistic number was estimated to be $7 \mathrm{~kg} / \mathrm{m}^{2} \pm$ $2 \mathrm{~kg} / \mathrm{m}^{2}$. The uncertainties in the ballistic number included uncertainties in mass, drag coefficient, and the actual reference area of the fully deployed shape.

Recall, that these missions maintained the fully deployed shape throughout the exoatmospheric phase of the flight and the shape was not modulated. Hence, ballistic number and the atmospheric profile mostly determined the flight trajectory. It was seen that the JB2008 model came closest to replicating the flight trajectory for TES3, although the ballistic number estimate had to be adjusted to $11.8 \mathrm{~kg} / \mathrm{m}^{2}$ to reconcile with the flight data. TES3 flight was undertaken well before higher-fidelity aerodynamic analysis was conducted; thus, the reconciliation suggesting a ballistic number slightly outside of the initial estimate is not unexpected. Reconciliation of the TES3 data was the major driver for selecting the JB2008 thermosphere model for the simulation. Similar comparison with the TES4 data showed best reconciliation of the flight data when using the JB2008 model and adjusting the ballistic number to $5 \mathrm{~kg} / \mathrm{m}^{2}$. Note, the vehicle between TES3 and TES4 were not the same size and shape, so the difference in ballistic numbers is justified.

\section{B. Effect of Drag Modulation and Atmospheric Variation}

Control authority of the Exo-brake is determined by the amount of drag modulation that can be achieved prior to $90 \mathrm{~km}$ when the REBR is released. Since control authority is key to landing accuracy and the current Exo-brake design is limited to reference length variation from 60 to 100\% (area variation of 76 to 100\%), it was not certain that this small variation in drag area would be sufficient to handle the large thermosphere dispersions to meet the $50 \mathrm{~km}$ desired target accuracy.

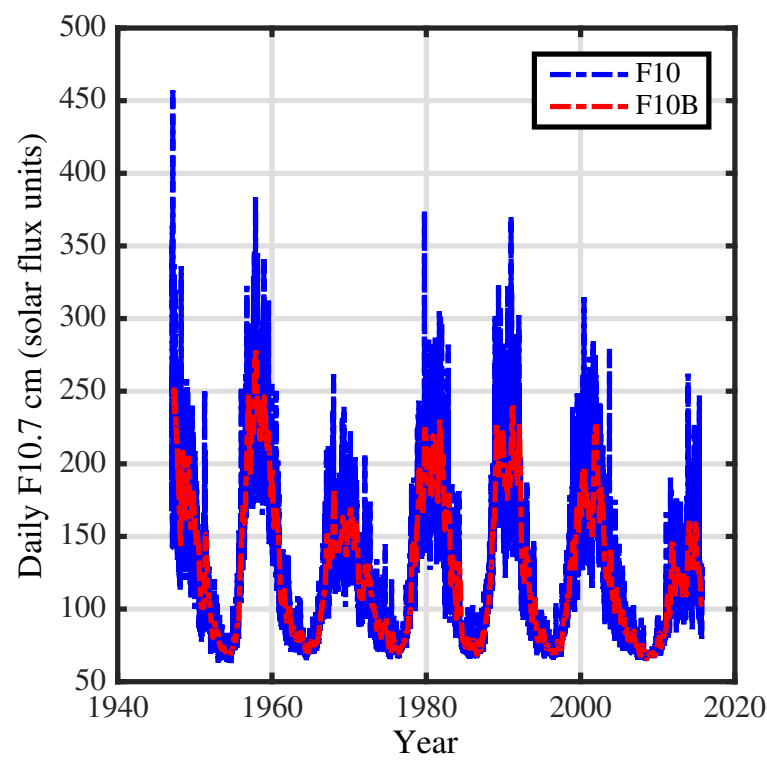

(a) Solar Radio Flux at $10.7 \mathrm{~cm}$ Variation ${ }^{12}$

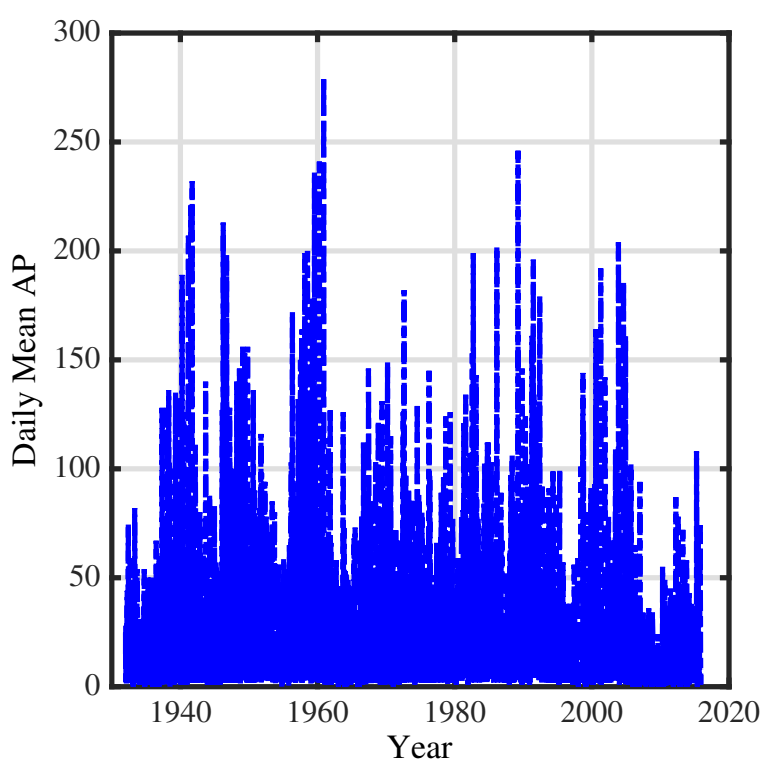

(b) Geomagnetic Index Variation ${ }^{13}$

Figure 7. Variation of exoatmospheric parameters over years.

In order to explore the problem, Fig. 7 shows the variation in F10.7 and AP data over the last century. It was noted that although over large time spans F10.7 and AP do vary by significant amounts, but over shorter time periods the values are more stable, as seen in the F10B (or the 81 day moving average) data. 
The values are also cyclical in nature and repeat every ten to twelve year coincident with solar maxima, as expected.

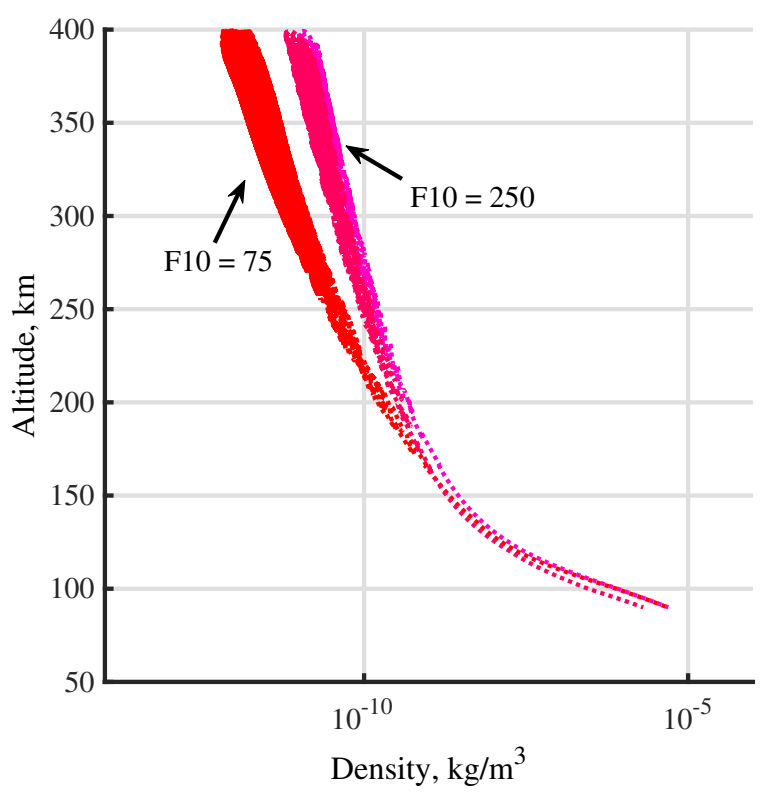

(a) Density Variation due to F10.7

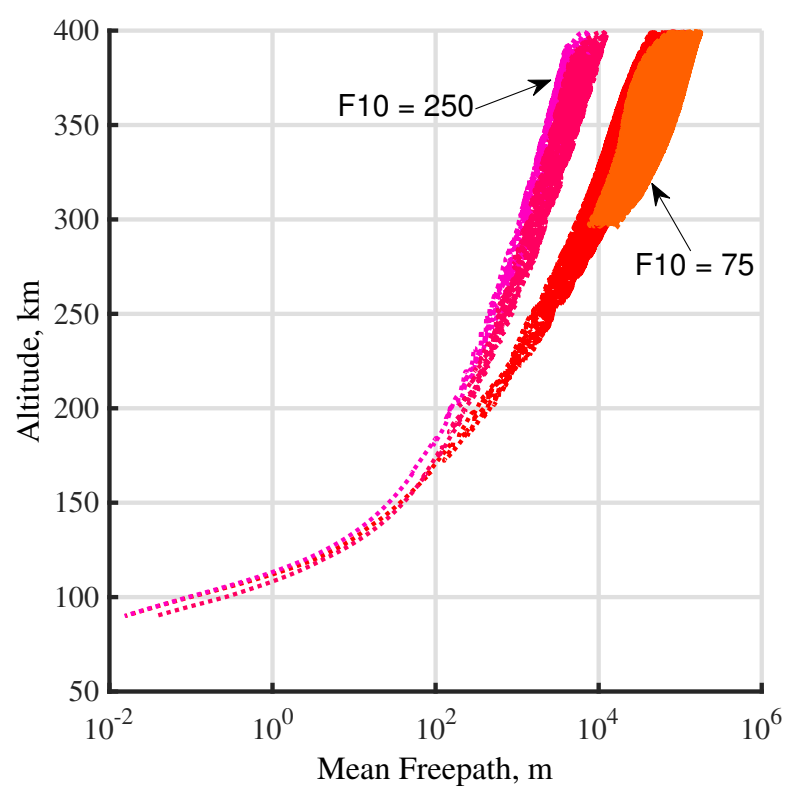

(b) Mean Freepath Variation due to F10.7

Figure 8. Atmospheric parameter variation due to Solar heat flux at $10.7 \mathrm{~cm}$ wavelength (F10.7) .

If the large F10.7 and AP variabilities are modeled in the JB2008 thermospheric model, there are indeed large dispersions in atmospheric parameters of interest, such as density and mean freepath, and this is shown in Fig. 8. In Fig. 8, F10.7 and AP are varied between their maximum and minimum value in the last 10 years and the Earth GRAM flag to simulate atmosphere within $\pm 3 \sigma$ were varied between the maximum and minimum values. The variations in atmospheric density and mean freepath were recorded. Not surprisingly, it was seen that one of the strongest drivers of density difference was the F10.7 term that split the density and mean freepath profiles into two families - lowest density and largest mean freepath are related to a small F10.7 while the highest density and lowest mean freepath are related to a larger F10.7. Note, for F10.7 and $\mathrm{AP}$, the variation was only between their maximum and minimum value. But the distinct families created in the figure were driven only by what F10.7 value was chosen.

Interestingly, the AP changes and Earth GRAM flag, which were also varied between their maximum and minimum value in the results shown in Fig. 8, only created very minor variations in atmospheric properties when compared to the variation caused by F10.7. Since data from Fig. 7 suggests that F10.7 does not change very rapidly within a short time period (of the order of Exo-brake's de-orbit trajectory), atmospheric variations due to the solar heat flux term may not be as big of a concern as thought earlier. In the simulation, the F10.7 and AP data from Fig. 7 are used to change the F10.7 and AP input to the thermosphere model as the trajectory is propagated in time.

The effects of the variation in some of these atmospheric parameters as well as the deployment level of the Exo-brake on the de-orbit trajectory are shown in Fig. 9. The simulation was run with a fixed deployment level throughout the flight for these cases. Initial concerns that the relatively small differential in reference area that occurs when the Exo-brake is modulated between $60 \%$ and $100 \%$ deploy levels were mollified when it was seen that there is at least eight days of difference in de-orbit time between trajectories flying one of the extrema of the deploy levels. If the difference in de-orbit time for flying a maximum deploy level case and minimum deploy level case was mere hours, that would have suggested that changing deployment level would do little to control the trajectory shape or course. However, eight days at least allows some leeway for the guidance to correct for off-nominal, unexpected behavior and still hit desired targets.

The difference between $60 \%$ deployment on a $-3 \sigma$ day (as defined by Earth GRAM and JB2008 model) and $100 \%$ deployment on a $+3 \sigma$ is the order of 18 days and that shows a reasonable range of time in which one may expect the guidance scheme for the Exo-brake to operate in and issue drag modulation commands to control the trajectory. This means that a drag modulation guidance scheme should be robust enough to 


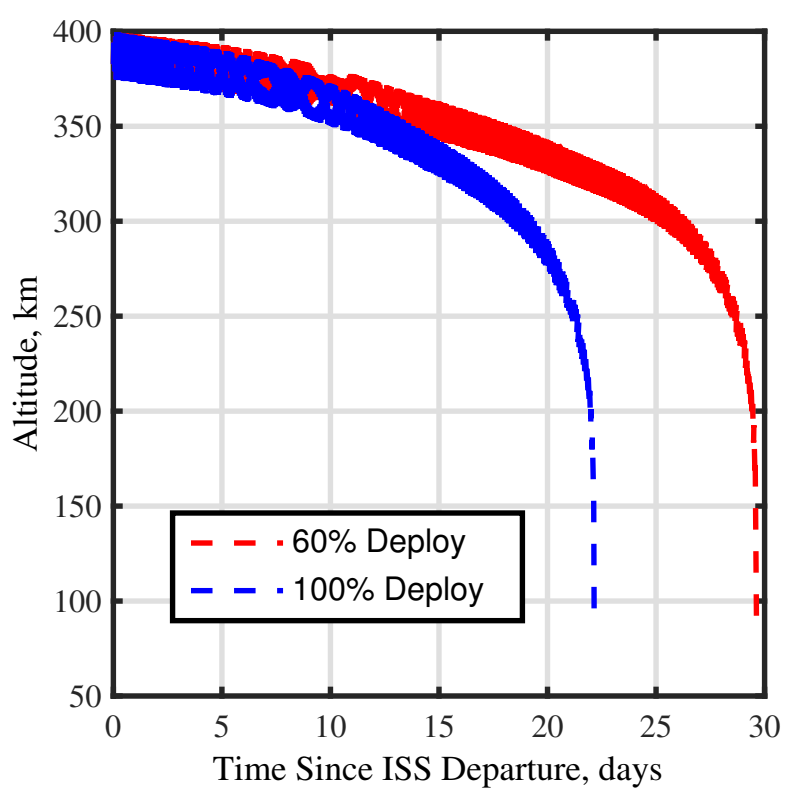

(a) Effect of Deployment Level

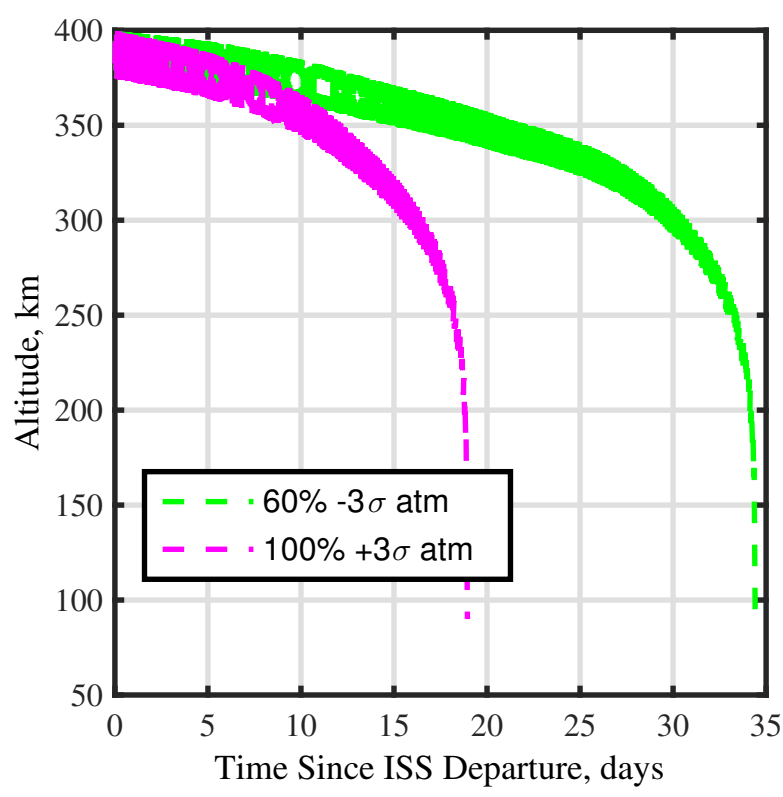

(b) Effect of Atmospheric Dispersion

Figure 9. Variation of de-orbit trajectories to vehicle and atmospheric parameters.

handle such atmospheric perturbations.

\section{Drag Modulation Guidance Scheme}

The guidance scheme that is discussed in this paper is a predictor-corrector type of algorithm where an end-to-end trajectory is simulated with a set of deployment time and levels, and then based on the cost function of the run, a projected-gradient based optimizer modifies the deployment time and level until the optimum is achieved within a tolerance. Since the goal of the problem being considered here is to land within a $50 \mathrm{~km}$ circle of UTTR, the cost function is the miss distance from the target. One of the tuning variables of this guidance scheme is to determine the number of deployment changes the Exo-brake can make during the flight. Operators of the Exo-brake spacecraft want to minimize the number of deployment changes to lower the risk of failure of the relatively untested modulation mechanism. Hence, continuously varying modulation schemes were highly discouraged. Additionally, the guidance scheme will be run off-line rather than onboard for the first few modulation Exo-brake flights. Some of these operational constraints initially suggested that guidance should be able to achieve desired targets with only a handful of deployment changes. For the results shown below, the guidance was allowed to change deployment levels only seven times during the flight. Recall, after jettison from ISS, the Exo-brake is held for approximately 5 days at the $100 \%$ deployment level and this is not included as one of the seven deploy level changes.

Figure 10 shows the result of a 1200 case Monte Carlo analysis where the Exo-brake vehicle followed drag modulation commands from the guidance scheme. As discussed before, atmospheric quantities and the REBR capsules aerodynamics quantities were varied in this Monte Carlo analysis. Hence, the predictorcorrector guidance scheme had to adjust for variable atmosphere and uncertainties in the aerodynamics. It can been seen from the landing points that the guidance scheme with seven deployment levels still had many cases that landed outside the $50 \mathrm{~km}$ circle around the target. Figure 11 shows that the $87 \%$ of cases landed within a $50 \mathrm{~km}$ radius of the target point. This underscores the fact that improvements are still required to tune the guidance so that a higher percentage of the cases reliably reach the $50 \mathrm{~km}$ proximity goal.

Figures 12 and 13 show box-and-whiskers plots that depict the various deployment levels and times chosen by the guidance scheme for the Monte Carlo cases. Considering the deployment times and values chosen by the guidance scheme during the Monte Carlo analysis, one notices a trend that early in the Exo-brake trajectory, the guidance chooses smaller deploy levels but slowly ramps up to near maximum deploy levels near the end of the trajectory. The saturation of the control authority near the end of the orbit suggest that the optimization problem is most sensitive to changes in deployment values near the end of trajectory, 


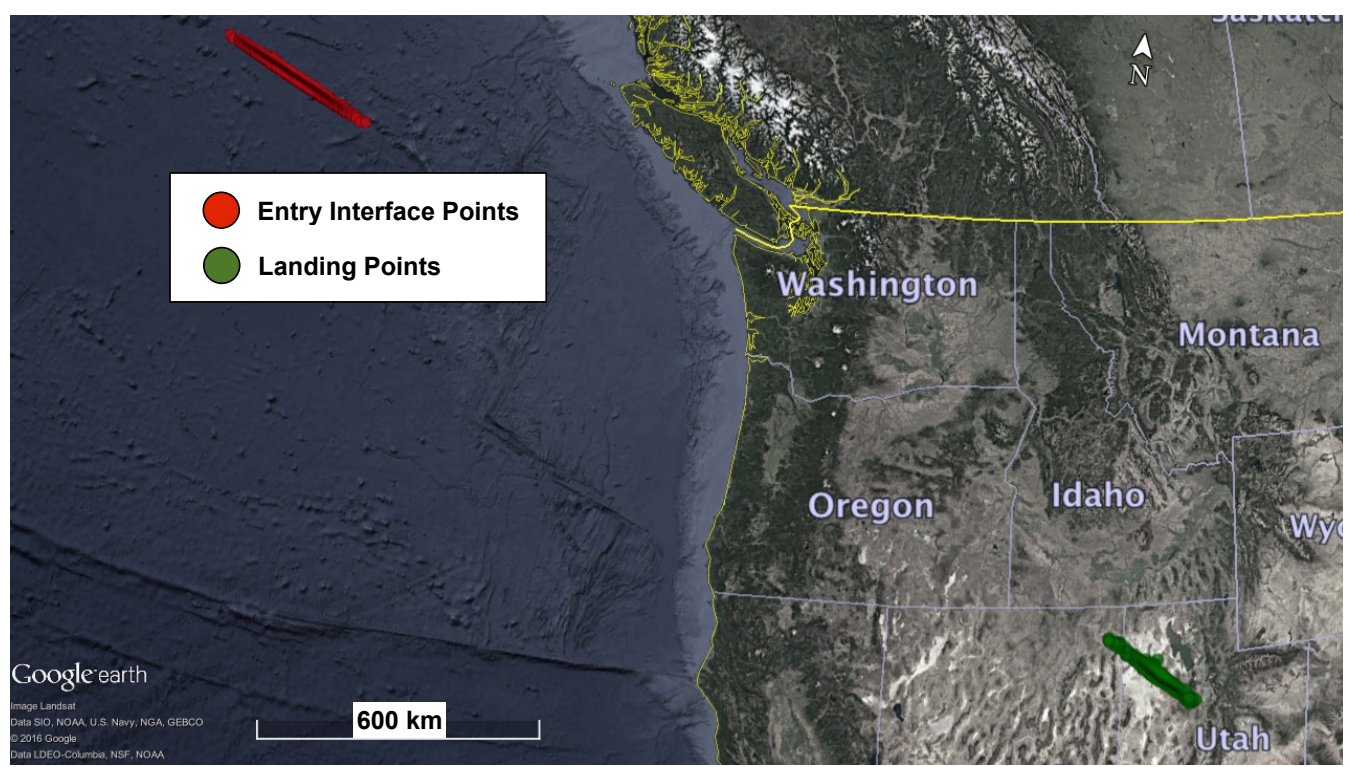

(a) Entry Interface and Landing Points

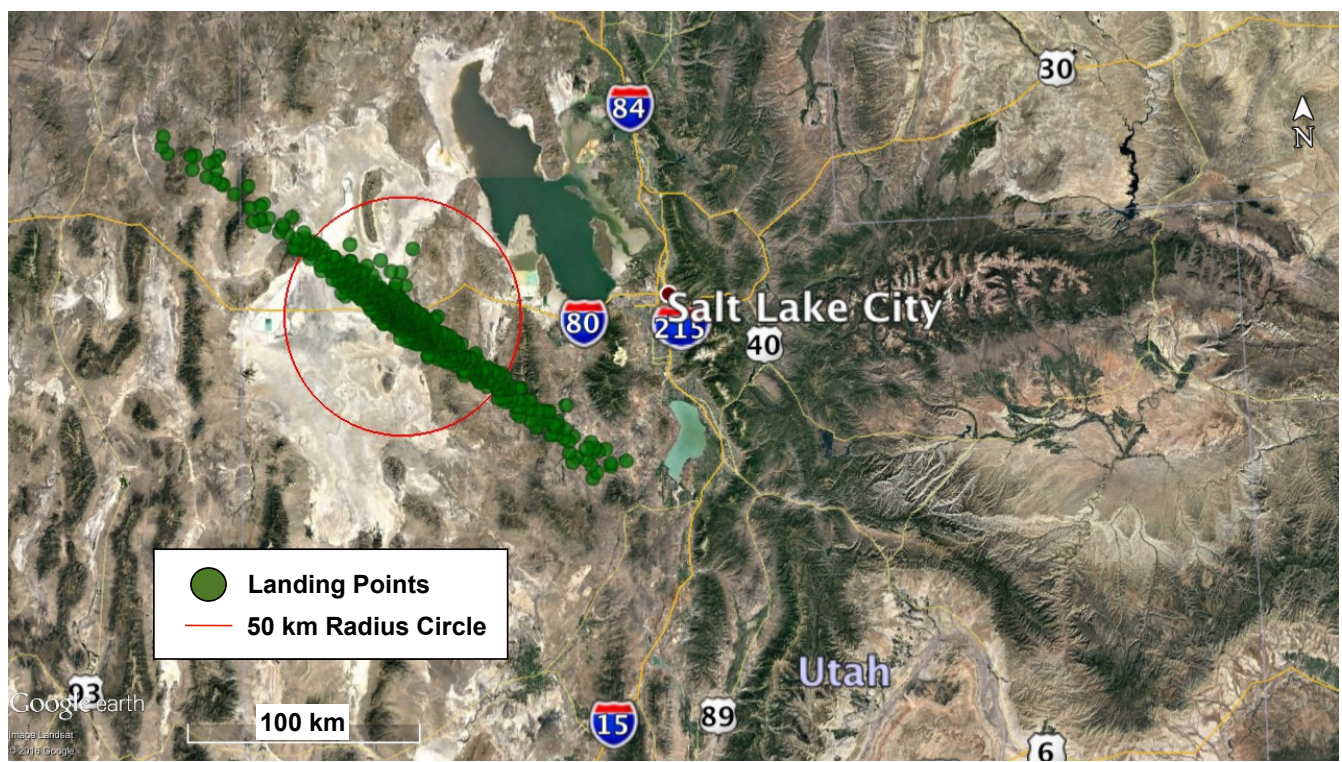

(b) Zoomed View of Landing Points

Figure 10. Landing Points from Monte Carlo analysis of the Exo-brake and REBR vehicle following drag modulation guidance.

which typically occur at lower altitudes and higher dynamic pressure areas. There is room for improvement in the guidance's performance by adding more deployment sequences near the end of the trajectory. Another observation from the box-and-whiskers plots is that the deployment levels and times for most of the cases are very congregated (hence the lack of distinction in each box-and-whisker). This Monte Carlo analysis had variations in atmospheric parameters and REBR aerodynamic quantities, but the guidance chose very similar deployment profiles in all of these random cases. Once aerodynamic uncertainties of the Exo-brake vehicle are available, these too will be varied in the Monte Carlo simulation, along with adding initial state variation to evaluate how timing deployments narrows the landing dispersions, modeling sensitivity to F10.7 variations, and switching to a six degree-of-freedom simulation for the Exo-brake. It is possible that with these changes in the future the guidance scheme would display wider variation in the commanded deployment history. 


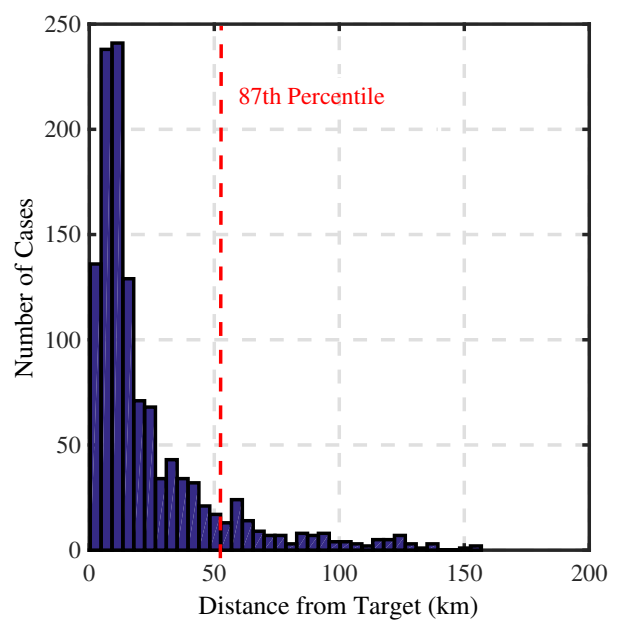

Figure 11. Histogram of miss distance from the target for the Monte Carlo cases.

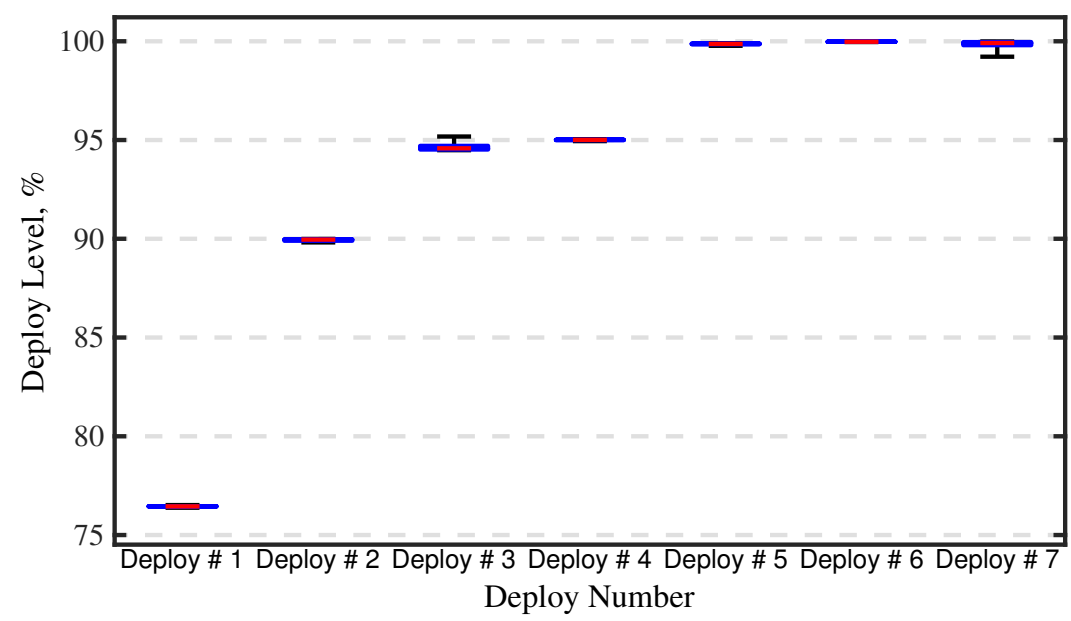

Figure 12. Box-and-whiskers plot of guidance commands for deployment levels from Monte Carlo analysis of the Exo-brake and REBR vehicle following drag modulation. Whiskers denote 5th and 95th percentile values, the box bounds 25th and 75th percentile values, and the red line in the middle denotes 50th percentile.

\section{Conclusion}

Passive drag based devices, such as the Exo-brake, allow payloads to be retrieved from assets in low Earth orbits, such as the International Space Station, without the need for onboard propulsion. These devices can be combined with small re-entry capsules, like the Reentry Breakup Recorder to safely land payloads on Earth. If the drag devices are modulated to provide differential drag area during their decaying orbit, one can improve the targeting of the landed location. This paper describes a simulation where the Exo-brake and REBR vehicles were modeled in three degree-of-freedom. Additionally, the performance of a predictorcorrector guidance scheme was described that modulates the Exo-brake only seven times during the orbit but can land a payload within $50 \mathrm{~km}$ of the target $87 \%$ of the time. However, the guidance scheme still needs to improve to lower the percent of cases that miss the target objective and the simulation needs to be updated to capture variation in the Exo-brake aerodynamics and potential drop off location from the International Space Station. 


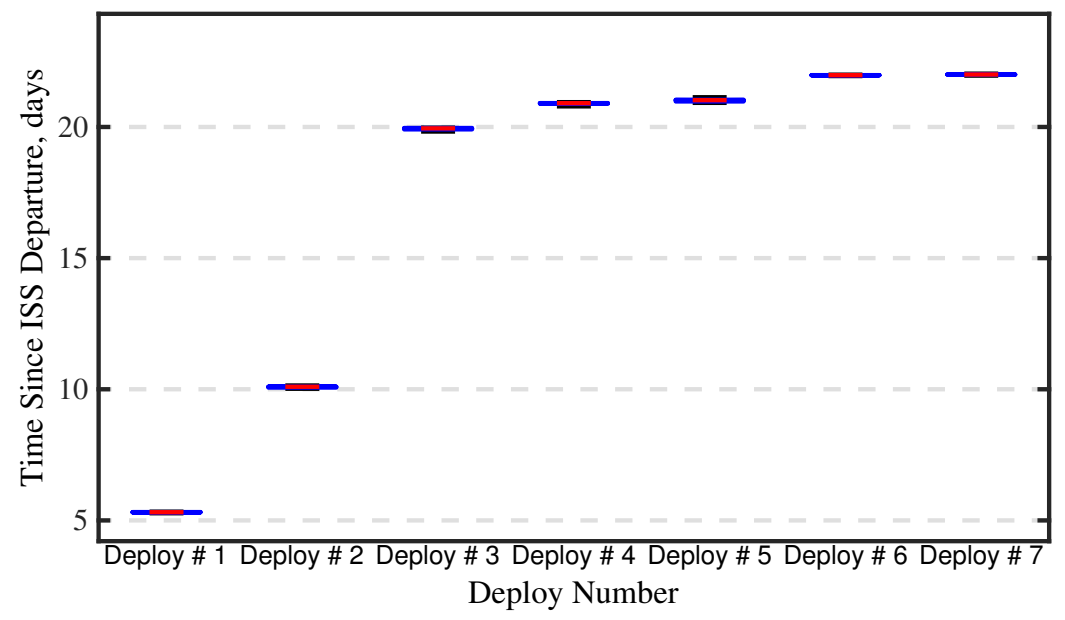

Figure 13. Box-and-whiskers plot of guidance commands for deployment times from Monte Carlo analysis of the Exo-brake and REBR vehicle following drag modulation. Whiskers denote 5th and 95th percentile values, the box bounds 25 th and 75 th percentile values, and the red line in the middle denotes 50th percentile.

\section{Acknowledgments}

The authors would like to acknowledge Marc Murbach of NASA Ames Research Center for his insight about the Exo-brake system. Additionally, the authors would like to thank Robert Dillman, from NASA Langley Research Center, for helping to estimate Exo-brake properties. This work was funded by the Entry Systems Modeling project under the Space Technology Mission Directorate of NASA. Michael Wright and Michael Barnhardt have supported the Exo-brake simulation team through those efforts.

\section{References}

${ }^{1}$ Murbach, M., Boronowsky, K., Benton, J., White, B., and Fritzler, E., "Options for RetReturn Payloads from the ISS after the Termination of STS Flights," AIAA 2010-6223, 40th International Conference on Environmental Systems, 2010.

${ }^{2}$ Leidich, J., Bannon, E., Leimkuehler, T., Chiesi, S., Mayer, N., and Boronowsky, K.Mand Murbach, M., "Options for RetReturn Payloads from the ISS after the Termination of STS Flights," AIAA 2011-7173, AIAA SPACE 2011 Conference 83 Exposition, Long Beach, CA, 2011.

${ }^{3}$ Murbach, M., "The TechEdSat NanoSatellites and Exo-Brake De-orbit Mechanism: Recent Flight Experience." LPI Contributions, Vol. 8120, 2014.

${ }^{4}$ Weaver, M. and Ailor, W., "Reentry Breakup Recorder: Concept, Testing, Moving Forward," AIAA 2012-5271, AIAA SPACE 2012 Conference \&S Exposition, Pasadena, CA, 2012.

${ }^{5}$ Braun, R. D., Powell, R. W., Engelund, W. C., Gnoffo, P. A., Weilmuenster, K. J., and Mitcheltree, R. A., "Mars Pathfinder Six-Degree-of-Freedom Entry Analysis," Journal of Spacecraft and Rockets, Vol. 32, No. 6, 1995, pp. 993-1000.

${ }^{6}$ Desai, P. N., Schoenenberger, M., and Cheatwood, F. M., "Mars Exploration Rover Six-Degree-of-Freedom Entry Trajectory Analysis," Journal of Spacecraft and Rockets, Vol. 43, No. 5, Sept. 2006, pp. 1019-1025.

${ }^{7}$ Desai, P. N., Qualls, G. D., and Schoenenberger, M., "Reconstruction of the Genesis Entry," Journal of Spacecraft and Rockets, Vol. 45, No. 1, Jan. 2008, pp. 33-38.

${ }^{8}$ Desai, P. N., Prince, J. L., Queen, E. M., Schoenenberger, M., Cruz, J. R., and Grover, M. R., "Entry, Descent, and Landing Performance of the Mars Phoenix Lander," Journal of Spacecraft and Rockets, Vol. 48, No. 5, 2011, pp. 798-808.

${ }^{9}$ Way, D. W., Davis, Jody, D., and Shidner, J. D., "Assessment of the Mars Science Laboratory Entry, Descent, and Landing Simulation," AAS 13-420, AAS/AIAA Space Flight Mechanics Conference, Kauai, HI, 2013.

${ }^{10}$ Mitcheltree, R., Moss, J., Cheatwood, F., Green, F., and Braun, R., "AAerodynamics of the Mars Microprobe Entry Vehicles," AIAA 1997-3658, 22nd Atmospheric Flight Mechanics Conference, New Orleans, LA, 1997.

${ }^{11}$ Leslie, F. and Justus, C., "The NASA Marshall Space Flight Center Earth Global Reference Atmosphere Model - 2010 Version," Tech. rep., NASA/TM-2011-216467, 2011.

12 "Solar Heat Flux at $10.7 \mathrm{~cm}$ Data," 2016.

13 "Geomagnetic Index (KP and AP) Data," 2016. 